\title{
Diderot et Maupertuis sur la sensibilité de la matière, ou comment feindre de ne pas se comprendre
}

\author{
Diderot and Maupertuis on the sensibility of matter, \\ or the practice of feigned misunderstanding
}

\author{
Michael A. Soubbotnik \\ Université Paris-Est Gustave Eiffel, LISAA EA4120 \\ Universidade Federal de Espírito Santo, PPGHis \\ michael.a.soubbotnik@gmail.com
}

\begin{abstract}
In $\S 50-51$ of his Pensées sur l'interprétation de la Nature, Diderot draws consequences adverse to religion from the hypothesis of the sensibility of living matter in the Systeme de la Nature, prompting an endangered Maupertuis to respond. Diderot knows that he is reading his own materialistic conception of matter in Maupertuis but is feigning to object to it, while Maupertuis, who knows that the so-called objection is in truth Diderot's own view, is compelled to defend himself. Through this rhetoric of feigned misunderstanding, real and fundamental questions (living matter, continuity, totality, unity of natural philosophy) raised by the phenomena of organization are being discussed.
\end{abstract}

Keywords: Diderot, Maupertuis, sensibility of matter, organization, continuity, totality

Diderot mène souvent la recherche philosophique dans et par le matériau littéraire et jusque dans la fabrique même d'un «style » dont George Daniel a pu dire que, par ses modalités dialogiques, « il ne fait que reproduire [...] les types de schématisation du réel » mécaniste vs organiciste qui dominent l'époque (Daniel, 1986, p. 35). Diderot va toutefois au-delà d'une telle reproduction dans ses «conjectures » les plus audacieuses, tout particulièrement quand elles touchent à la périlleuse question du matérialisme. Le dialogue peut alors perdre de ce qui lui restait d'innocence 
ludique. La rhétorique de l'invention doit ruser avec la censure et se faire pratique du déplacement et/ou du travestissement. Un cas exemplaire et fondamental est celui des paragraphes 50 et 51 des Pensées sur l'interprétation de la nature ${ }^{1}$ (Diderot, 1875b [1754], p. 45-50), ce « texte-paradigme » (Saint-Amand, 1984, p. 23) qui mène Diderot sur les voies d'une pensée de la complexité ${ }^{2}$. Cas fondamental que ces paragraphes, d'abord par la présence de ce «leibnizo-newtonianisme » où Anne-Lise Rey voit à juste titre, relisant Maupertuis et Émilie du Châtelet, l' « enjeu de la construction d'une philosophie naturelle complexe » (Rey, 2003) ; ensuite parce que Diderot, en accompagnant et, dirait-on, en dévoyant Maupertuis dans la pensée de cette complexité, pose, dès 1754 , les prémisses de ce qui constituera sa pensée définitive concernant la matière partout vivante. C'est que Maupertuis incarne l'entrecroisement des quatre questions qui surgissent dans les PIN et que le Rêve de d'Alembert tentera de résoudre : l'organisation, la génération, la relation inerte/vivant, l'unité (du vivant, de la vie, du tout, du moi, etc.) (Ibrahim, 2003, p. 73). Cas exemplaire aussi, d'abord parce qu'il le fait à partir d'une lecture de l'hypothèse de la sensibilité des éléments matériels, que le Dr Baumann, alias Maupertuis, vient d'exposer dans son Système de la Nature ${ }^{3}$, principalement aux paragraphes 51 à 58 (Maupertuis, 1756b [1754], p. $154 *-159 *^{4}$ ) ; ensuite parce qu'il présente cette hypothèse comme objet d'une réfutation par un passage à la limite qui consiste, en réalité, à en adopter la généralisation à l'Univers entier. Parce que cette déduction des conséquences extrêmes de l'hypothèse assimile sa conjecture à une thèse matérialiste dangereuse pour la religion, Maupertuis se voit dans l'obligation de publier une Réponse aux Objections de M. Diderot.

La lecture du texte de Diderot et de cette réponse révèle, en parcourant le labyrinthe de leur rhétorique de feinte et mutuelle mécompréhension, que les questions cruciales du continu et du tout sont au cœur de l'enjeu, perçu de manière bien différente par le mathématicien et par le philosophe, d'une possible réunification du domaine de la philosophie naturelle après l'échec du mécanicisme face au vivant.

Dans le $\S 50$ des $P I N$, l'examen de l'hypothèse d'une matière sensible consiste en un travail de passage à la limite par changement d'échelle. D'une part, en faisant apercevoir «les terribles conséquences» de l'hypothèse, Diderot détermine les

\footnotetext{
${ }^{1}$ Désormais abrégé en $P I N$.

${ }^{2}$ Pour les divers aspects de la pensée diderotienne de la complexité, voir tout l'ouvrage de Saint-Amand (1984).

${ }^{3}$ Désormais abrégé en $S N$.

${ }^{4}$ Nous reproduisons l'astérisque dont l'édition de 1756 marque certaines pages numérotées en doublet.
} 
enjeux d'une attribution de la sensibilité à la matière pour la science de l'époque dans son rapport à la religion. D'autre part, il inverse le changement d'échelle opéré par Maupertuis qui passait de la sensibilité de l'animal à celle de la particule de matière vivante ; Diderot (1875b [1754], p. 48), lui, tire de la sensibilité de l'animal celle de l'univers entier.

Ce travail répond aux préoccupations épistémologiques qui caractérisent l'ensemble des PIN. Ainsi Diderot (1875b [1754], p. 48-49) oppose-t-il deux types de conjectures ou d'hypothèses à la fin du paragraphe : celles du métaphysicien, qui débouchent sur des généralisations abstraites, et celles du physicien, destinées au contrôle de l'observation et de l'expérience ${ }^{5}$. Or, ce n'est pas tout-à-fait ainsi que Maupertuis envisageait les hypothèses. Rien dans le $S N$ n'indique en effet qu'il soit revenu sur l'essentiel du projet de l'Essai de Cosmologie de $1751^{6}$. À l'opposé de Réaumur (1734, p. 2), pour qui «l'immensité des ouvrages de la nature ne paraît mieux nulle part que dans l'innombrable multiplicité » des phénomènes, il s'agissait alors de rechercher les preuves de l'existence de Dieu « dans les premières lois qu'il a imposées à la Nature ; dans ces règles universelles selon lesquelles le mouvement se conserve, se distribue, ou se détruit » (Maupertuis, 1756a [1751], p. 23). Rien n'indique non plus que Maupertuis ait cessé en 1754 de considérer que la suprême Intelligence transcende les lois qu'elle « impose » à la Nature. Maupertuis et Diderot ne situent manifestement pas la généralisation sur le même registre. Les hypothèses métaphysiques que Diderot assigne à l'ontologie et à la théorie de la connaissance, Maupertuis les renvoie : soit à une théologie devant laquelle il s'incline (à condition qu'elle se cantonne au domaine de l'origine et des fins dernières), soit aux spéculations systématiques (de Malebranche, Leibniz ou Spinoza) qu'il récuse. Quant aux hypothèses physiques, il cherche en newtonien à leur accorder la généralité maximale compatible à une mise à l'épreuve par l'expérimentation ou par l'observation.

Cette différence de conception épistémologique se retrouve dans le sens donné à la notion de « système ». Au début du $\S 50$, Diderot écrit que l'hypothèse porte sur le système de la nature. Simple reprise du titre de l'œuvre de Maupertuis ? Condillac (1947 [1749], p. 121) écrit, de manière très générale, au début de son Traité des Systèmes, qu'un système « n'est autre chose que la disposition des différentes parties d'un art ou d'une science dans un ordre où elles se soutiennent toutes mutuellement, et où les dernières s'expliquent par les premières ». Où l'on voit que toute la question est de savoir à partir de quels principes on établit cet ordre. À cet effet, Condillac (1947 [1749], p. 121) explique immédiatement qu'il y a trois sortes de principes, donc de systèmes : les systèmes «abstraits » dont les principes sont des

\footnotetext{
${ }^{5}$ Nous avons traité ailleurs du rapport du spéculatif et de l'expérimental dans une rhétorique de l'invention chez Diderot (Soubbotnik, 2010).

${ }^{6}$ Pour plus de détails voir Roger (1993 [partie III, chapitre 1]) et la discussion menée par Fichant (1975).
} 
« maximes générales ou abstraites» comme, par exemple, le principe de contradiction; les «hypothèses [...] qui n'ont que des suppositions pour fondements » et que Condillac compare aux rêves; les «vrais systèmes », enfin, fondés sur l'expérience et l'observation et dont le système de Newton est le modèle. Il semble que Maupertuis envisage son système de la Nature dans ce troisième sens ; Diderot, lui, rangerait l'hypothèse de Maupertuis dans la seconde catégorie, qu'il feint ici de dénoncer pour, dans la trilogie de 1769, assumer sous la forme d'un rêve de d'Alembert, les « terribles conséquences » de la version qu'il en avait donnée en 1754.

Le problème posé dans les PIN est donc bien celui de la rationalité et de la positivité de suppositions dont l'objet (le vivant) semble devoir échapper aux lois du système de la physique newtonienne. Peut-on transposer des lois d'un ordre de phénomènes à un autre (la loi d'attraction, par exemple, de la physique à la chimie) ? Quelles transformations les lois subissent-elles au cours de la transposition? Une fois la transposition accomplie, peut-on garantir ou rétablir une unité d'interprétation des phénomènes naturels? Là où l'expérience et l'observation risquent de faire défaut, est-il possible de forger des suppositions rationnelles qui ne soient pas que des idées abstraites? Y a-t-il, enfin, une unité de la nature, un « Tout» de la nature auquel pourrait répondre une unité de la science ? En quel sens et à quel prix suppose-t-on cette unité ?

Tout en se voulant rattachée à l'expérience, l'hypothèse de Maupertuis n'en est pas pour autant vérifiable par l'expérimentation ou l'observation. Certes, la sensibilité s'observe chez l'animal (d'où le préalable consistant à récuser la thèse de l'animal-machine) mais l'attribuer à l'élément est changer l'ordre des grandeurs pour passer du grand observable au petit qui ne l'est plus. Cela autorise-t-il Diderot à voir chez Maupertuis les prémisses d'un changement d'ordre inverse, aux conséquences « terribles »? Maupertuis le nie absolument et il convient maintenant d'interroger sa réponse pour entrer dans les détours de la controverse.

\section{III}

La Réponse aux objections de Monsieur Diderot prétend s'inscrire dans un cadre classique de controverse philosophique. Mais s'agit-il bien d'objections dans le $\S 50$ des PIN ? Certes, l'incipit de Diderot annonce que « pour ébranler une hypothèse, il ne faut quelquefois que la pousser aussi loin qu'elle peut aller » (Diderot, 1875b [1754], p. 45). Mais le passage à la limite par changement d'échelle de la molécule au Tout est plutôt destiné à reconstruire la conjecture qu'à l'ébranler. Quoi qu'il en

\footnotetext{
${ }^{7}$ Pour une analyse fine de la manière dont Diderot, dans l'ensemble de son œuvre, aborde ces problèmes en relation aux sciences de la nature de son temps, voir par exemple Guédon (1979), Ibrahim (1999), Bourdin (2003), Quintili (2006) et pour une étude étendue à l'Encyclopédie elle-même, voir, par exemple, Lojkine (1999).
} 
soit, Maupertuis (1756b [1754], p. 169-170 et 171) se voit «obligé de répondre » à des objections «sur lesquelles le silence serait une faute ou contre la société ou contre la foi » et auxquelles s'ajoutent «des réflexions capables de mortifier ou d'alarmer l'auteur ». À un Diderot qui fait passer pour une objection ce qui est sa propre construction visant soit à éliminer Dieu, soit à l'identifier à la Nature, Maupertuis, qui n'a jamais cessé de contester explicitement que l'édifice, bien qu'autonome, de la philosophie naturelle puisse se passer d'une Intelligence transcendante, se voit précisément obligé de répliquer comme si l'objection en était vraiment une parce que la protection dont Diderot s'entoure le met, lui, en péril. Pour autant, Maupertuis n'est pas dupe : «Si l'on était moins persuadé de la religion de l'Auteur de l'interprétation de la Nature », écrit-il ironiquement, " on pourrait soupçonner que son dessein n'est pas tant de détruire l'hypothèse, que d'en tirer ces conséquences qu'il appelle terribles » (1756b [1754], p. 171). Chacun comprend donc parfaitement l'autre, mais feint de le mécomprendre en le prenant au mot soit de ce qu'il lui attribue, soit de ce qu'il prétend faire. L'un prêtant à l'autre les mots que lui-même n'avouait pas penser, l'autre prêtant à l'un de ne pas penser ce qu'il lui prêtait, les deux auteurs jouent au jeu, au total fécond, des « objections et réponses ».

Avant d'entrer dans le détail des objections, Maupertuis en conteste la méthode. $\mathrm{Y}$ a-t-il, demande-t-il, « quelque hypothèse philosophique dont on ne puisse si l'on veut tirer des conséquences terribles? » (Maupertuis (1756b [1754], p. 166*) : la méthode frappe trop large. Répliquera-t-on qu'elle n'a pas pour but de combattre l'hypothèse elle-même mais le système dont elle est le principe ? La méthode frappe alors trop court puisqu'elle suppose entre l'hypothèse princeps et ses conséquences une chaîne de raisons dont la consistance est purement illusoire : «Notre esprit, aussi borné qu'il est, trouvera-t-il aucun système où toutes les conséquences s'accordent ? Un tel système serait l'explication de tout : et croit-on jamais y parvenir?» (Maupertuis (1756b [1754], p. 167*). C'est bien pourquoi l'Essai de Cosmologie s'en tenait aux lois les plus générales conformes à l'observation et l'expérience. Nos systèmes les plus étendus «n'embrassent qu'une petite partie du plan » divin et « nous ne voyons ni le rapport des parties entre elles, ni leur rapport avec le tout » (Maupertuis, 1756b [1754], p. 167*). Reste à s'entendre sur ce que l'on appelle « voir ». « Voir les rapports " signifie autre chose pour Maupertuis que les rêver, les imaginer ou les délirer comme le fera Diderot qui, tout en préservant la rationalité stricte de l'état présent des sciences, se projette dans un au-delà temporel non transcendant où la cartographie des savoirs serait radicalement modifiée. Voir un rapport serait, en somme, pour Maupertuis concevoir une règle rendant compte, dans sa généralité régionale, d'un ensemble de phénomènes du même ordre et inscrire cette règle dans un langage adéquat à une philosophie naturelle qui, épurée de sa part métaphysique, préserverait, mais hors d'elle, la part théologique de la pensée humaine. On pourrait dire qu'à l'idée d'une chaîne continue de raisons à partir d'une hypothèse princeps, Maupertuis oppose la discontinuité fondamentale des connais- 
sances et une rupture toujours possible de la chaîne des raisons une fois qu'elle est sortie des limites de l'expérience et de l'observation. Cela définit le cadre dans lequel il formule sa fameuse hypothèse de la sensibilité des éléments naturels, ainsi que l'enjeu principal de la controverse telle qu'il la conçoit : l'alternative entre continuité et contiguïté.

\section{IV}

Dans le $\S 50$ du $S N$, Maupertuis (1756b [1754], p. 154*) suppose, contre le continuisme de Buffon (1884-1885 [1749], p. 43, 50-51, 92-94, 104-105) et de Diderot (1875b [1754], p. $16 ; \S 12)$, qu'après un cataclysme, de nouvelles combinaisons d'éléments produiraient (ou ont produit) des êtres vivants complètement différents. Dans le $\S 51$, cette supposition est mise au service d'une explication de notre incompréhension devant nos présentes observations, les « vestiges » de la « formation » des corps ayant été effacés pour laisser place à une vision du « tout » qui n'est que l'absence de vision du détail. Et Maupertuis de mobiliser cette métaphore de l'essaim - peut-être empruntée à Bordeu (1818 [1751], p. 187 ; § CXXV) - que Diderot (1875a [1769], p. 126) reprendra à d'autres fins dans le Rêve de d'Alembert: « C'est ainsi [...] qu'un essaim d'abeilles, lorsqu'elles se sont [...] unies autour de la branche de quelque arbre, n'offre plus à nos yeux qu'un corps qui n'a aucune ressemblance avec les individus qui l'ont formé ») (Maupertuis, 1756b [1754], p. $\left.154^{*}-155^{*}\right)$. Dans l'usage que Maupertuis fait de la métaphore, l'essaim n'est une unité qu'en raison de l'éloignement de notre regard. C'est bien pourquoi Diderot se verra contraint, pour obtenir une métaphore de l'unité par continuité, d'amollir, par expérience de pensée, les pattes des abeilles de manière à établir entre ces «éléments » une véritable continuité ${ }^{8}$.

Le contenu de ces deux paragraphes 50 et 51 du $S N$ constitue le soubassement de l'hypothèse que Maupertuis reproche à son adversaire de travestir. Dans les paragraphes incriminés (p. 52-54), une fois que les éléments ou composants ultimes de la matière ont été dotés de sentiment ou perception, la question est de savoir ce qu'il advient lorsque ces éléments s'unissent pour former un corps organisé : «chaque élément, en déposant sa forme et s'accumulant au corps qu'il va former, déposeraitil aussi sa perception ? perdrait-il, affaiblirait-il le petit degré de sentiment qu'il avait; ou l'augmenterait-il par son union avec les autres, pour le profit du tout?» (Maupertuis, 1756b [1754], p. 155*). Toute réponse un tant soit peu détaillée à cette question devra reposer sur une « loi de la conservation de la perception », analogue à un principe de conservation des forces vives ${ }^{9}$. Puisque la perception est une «pro-

\footnotetext{
${ }^{8}$ Pour une analyse de ces métaphores dans le Rêve, voir Saint-Amand (1984, p. 43-49), Bourdin (2003, p. 54-56, 59-60), Ibrahim (2003) et Vuilllemin (2010).

${ }^{9}$ À partir du point de vue d'Émilie du Châtelet, Anne-Lise Rey (2003) a bien montré les enjeux philosophiques du principe de conservation.
} 
priété essentielle » des composants ultimes de la matière vivante, leur permettant de se combiner, elle peut se transformer selon les combinaisons mais non « périr, diminuer ni s'accroître ». Elle doit donc toujours « dans l'Univers, former une même somme, quoique nous ne puissions ni la suivre ni la reconnaître » (Maupertuis, $1756 \mathrm{~b}$ [1754], p. 155*). On perçoit aussitôt comment une telle généralisation aux « espèces différentes de la nôtre », qui ne peut s'opérer, comme Maupertuis le reconnaît, que par analogie avec «l'expérience de ce qui se passe en nous-mêmes » (1756b [1754], p. 156*), peut induire Diderot à passer, moyennant un principe de continuité qui double la loi de conservation de la perception, de l'élément à l'animal et de l'animal au Tout de l'Univers. Or, d'une part, Maupertuis ne parle expressément que des espèces vivantes et, d'autre part, leurs éléments ne contribuent pas également à la perception unique résultante puisque certains « assemblages » contiennent « les éléments dont les perceptions font la pensée » et que dans d'autres « il ne paraît aucune réunion de perceptions élémentaires qui puisse former pour nous aucun genre de perception » (1756b [1754], p. 157).

Alors que Diderot prend la conjecture pour un «principe» dont dépend tout le système de la nature et qui soit ébranlerait l'existence de Dieu, soit confondrait Dieu et la Nature, Maupertuis (1756b [1754], p. 171) répète que sa conjecture est tout à fait indépendante de la question du « système physique de la formation des corps » et qu'elle n'a aucune des conséquences que Diderot lui attribue sur la religion. La question est évidemment de savoir en fonction de quels arguments Diderot interprète la proposition de Maupertuis comme il le fait. Que l'on touche à un moment décisif du $§ 50$ des PIN, Diderot l'annonce lui-même : «C'est ici que nous sommes surpris que l'auteur, ou n'ait pas aperçu les terribles conséquences de son hypothèse, ou que, s'il a aperçu les conséquences, il n'ait pas abandonné l'hypothèse » (Diderot, 1875b [1754], p. 48). Le poids de la controverse se porte désormais sur la notion de « Tout».

Diderot tente d'enfermer Maupertuis dans un dilemme. L'Univers (pas le seul monde vivant) est défini comme «la collection générale de toutes les molécules sensibles et pensantes ». Cette collection « forme un tout ou non ». Si Maupertuis conteste que l'univers forme un tout, il introduit « le désordre dans la Nature », mettant ainsi en péril et l'existence de Dieu et les fondements de la philosophie naturelle «en rompant la chaîne de tous les êtres ». Si par contre il admet que l'Univers forme un tout, cela signifie, selon Diderot, que les «éléments [n'y] sont pas moins ordonnés que les portions, ou réellement distinctes, ou seulement intelligibles, le sont dans un élément, et les éléments dans un animal ». Or, cette caractérisation implique, pour Diderot, que « le monde, semblable à un grand animal, a une âme ; que, le monde pouvant être infini, cette âme du monde, je ne dis pas est, mais peut être un système infini de perceptions, et que le monde peut être Dieu » (Diderot, 1875 b [1754], p. 48).

Le dilemme est construit sur l'ambiguïté du mot «Tout». Bien que le terme reste, comme le note Maupertuis, «vague » dans la branche négative du dilemme, 
on peut y repérer deux sens assez distincts. En un premier sens, que le monde forme un tout est une condition nécessaire pour qu'il soit ordonné. En un second sens, que le monde forme un tout est une condition nécessaire pour que les êtres forment une chaîne continue. On peut donc supposer que la continuité de la chaîne des êtres est une condition nécessaire de l'ordre. Qu'en est-il alors du tout ? Est-il une continuité ordonnée ou bien quelque chose dont l'ordre et la continuité sont les conditions nécessaires et suffisantes?

Diderot semble plus précis dans la branche positive de l'alternative, sur laquelle pèse le poids de son objection. Le sens requis est celui qui ferait de la Nature un corps organisé par une âme du monde. Toute ambiguité n'est pourtant pas levée car le point de contention réside moins dans la réponse négative ou positive à la question que dans le sens et la portée que l'on attribuerait à une réponse positive. Maupertuis (1756b [1754], p. 174*) se refuse en effet à admettre que du fait que «les perceptions élémentaires conspirent à former une perception unique » dans certains corps comme ceux des animaux, il s'ensuive «que cette copulation de perceptions s'étende à l'univers entier ».

Nous voici ramenés à la question du continu, la continuité étant requise pour que le passage à la limite proposé par Diderot ait quelque légitimité. Il faut en effet qu'il y ait « continuité » de l'élément à l'animal et de l'animal à l'Univers, que continuité d'élément à élément ainsi que continuité de conjecture à conséquence. Il faut surtout, que le terme de «continu» garde un sens dans tous ces usages. S'il s'agit de faire «de la totalité de la matière un seul bloc, une seule pièce, un continu sans aucune interruption entre les parties », on objectera à Diderot l'existence du vide (1756b [1754], p. 174*). Or, même dans une pensée pléniste, « la différence entre ce continu et le disséminé ne consistera que dans le plus ou le moins de distance entre les parties, ne sera que l'effet de cette distance sur nos sens » (1756b [1754], p. 175*) - reprise de l'argument adossé à l'image de l'essaim.

Diderot ne nie évidemment pas le vide. Le tout dont il est question n'est pas celui d'une continuité de la matière dont Diderot (1875b [1754], p. 56 ; § 58) affirme d'ailleurs clairement l'hétérogénéité, mais «le Dieu de Spinoza » que Maupertuis récuse catégoriquement (1756b [1754], p. 175). Maupertuis sait fort bien, d'ailleurs, que la continuité dont Diderot se réclame, au risque, sans doute, d'abuser du terme, désigne tant une absence de séparation essentielle entre les règnes que la possibilité d'ampliation de l'hypothèse jusqu'au Tout de l'Univers. Et, parce qu'il le sait, Maupertuis conclut la controverse par une réponse au $\S 51$ des $P I N$, une coda où les deux adversaires semblent jouer à fronts renversés.

Diderot y suggère que si Maupertuis n'avait «appliqué ses idées qu'à la formation des animaux, sans les étendre à la nature de l'âme », il n'aurait pas cédé à la tentation du matérialisme. Diderot suppose à Maupertuis d'avoir attribué toutes les propriétés de l'âme, désir, aversion, sentiment, pensée, aux molécules organiques au lieu de se contenter de leur accorder une sensibilité sourde «mille fois moindre que 
celle que le Tout-Puissant a accordée aux animaux les plus voisins de la matière morte » (Diderot, 1875b [1754], p. 49). Diderot peut alors avancer sa propre définition de l'animal comme « un système de différentes molécules organiques qui, par l'impulsion d'une sensation semblable à un toucher obtus et sourd que [le créateur] leur a donné, se sont combinées jusqu'à ce que chacune ait rencontré la place la plus convenable à sa figure et à son repos » (1875b [1754], p. 49-50).

En somme, objecte Maupertuis, Diderot rompt la continuité entre la sensation et la perception pour substituer une différence de nature là où n'existe qu'une différence de «perfection ». Maupertuis continuiste ? Oui, s'agissant de ce qui admet continuité, comme la perception (qu'il prend évidemment dans un sens proche de celui de Leibniz, incluant les «sensations sourdes »). Mais voici, surtout, Diderot accusé d'un dualisme qu'il ne pourra véritablement congédier qu'en assumant pleinement, dans la trilogie du Rêve, les « conséquences » qu'il attribue ici à Maupertuis à travers la construction d'un modèle tout à fait nouveau, celui des cordes vibrantes, emprunté à d'Alembert (Diderot, 1875a [1769], p. 111-117). Car, comme Maupertuis prend un malin plaisir à le souligner, celui qui refuse la perception à la matière se condamne au bi-substantialisme. Mais qu'est-ce que cette «pensée » que le dualisme veut exclure de l'étendue ? Et de répondre presque comme le fera Diderot quelques années plus tard, qu'il s'agit « de la pensée en général, [...] la simple faculté d'apercevoir ou de sentir; [...] la sensation la plus obscure et la plus sourde » (1756b [1754], p. 181). Or, cette sensation-là est « une vraie perception » qu'il ne faut pas confondre toutefois avec les «perceptions claires et distinctes de notre âme » (1756b [1754]), p. 182 et 183). La continuité qualitative que l'on peut supposer entre les perceptions sourdes et les perceptions claires et distinctes ne contredit pas la discontinuité des règnes et des savoirs une fois que l'on est passé des lois les plus générales aux lois plus particulières régissant les différents règnes.

Les paragraphes 50 et 51 des PIN auront contraint Maupertuis à préciser les rapports complexes entre sa philosophie et sa théologie naturelles en les articulant à une distinction des domaines scientifiques. Diderot, lui, sort de cette controverse lesté, non seulement de la piste que lui a ouverte l'hypothèse de Maupertuis, mais encore de problèmes dont la résolution exigera, comme en témoigne le Rêve de d'Alembert, de nouvelles formes d'écriture et de nouvelles analogies. 


\section{BIBLIOGRAPHIE}

Bordeu, Th. de (1818 [1751]). Recherches anatomiques sur la position des Glandes et leur action. In Euvres complètes. T. 1. Paris : Caille \& Ravier.

Bourdin, J.-C. (2003). Du Rêve de d'Alembert aux Éléments de Physiologie. Discours scientifique et discours spéculatif dans le Rêve de d'Alembert. Recherches sur Diderot et sur l'Encyclopédie, 34, 45-69.

Buffon (1884-1885 [1749]). Histoire et Théorie de la Terre. In Euvres Complètes. T. I (pp. 34-414). Paris : A. Levasseur.

Condillac (1947 [1749]). Traité des Systèmes. In Euvres philosophiques. T. I (pp. 119-217). Éd. G. Le Roy. Corpus Général des Philosophes Français. Paris : P.U.F.

Daniel, G. (1986). Le style de Diderot. Légende et structure. Paris \& Genève : Librairie Droz.

Diderot, D. (1875a [1769]). Entretien entre d'Alembert et Diderot, Le rêve de d'Alembert, Suite de l'entretien. In Euvres Complètes. T. II (pp. 101-192). Éd. J. Assézat. Paris : Garnier.

Diderot, D. (1875b [1754]). Pensées sur l'interprétation de la Nature. In Euvres Complètes. T. II (pp. 1-53). Éd. J. Assézat. Paris : Garnier.

Fichant, M. (1975). Téléologie et théologie physique chez Maupertuis. In Actes de la journée Maupertuis (pp. 141-163). Éd. Olivier Bloch. Paris : Vrin.

Guédon, J.-C. (1979). Chimie et matérialisme. La stratégie anti-newtonienne de Diderot. Dixhuitième siècle, 11, 185-200.

Ibrahim, A. (1999). Le matérialisme de Diderot: formes et forces dans l'ordre des vivants. In Diderot et la question de la forme (pp. 87-103). Éd. A. Ibrahim. Paris : PUF.

Ibrahim, A. (2003). Maupertuis dans Le Rêve de D'Alembert : l'essaim d'abeilles et le polype. Recherches sur Diderot et sur l'Encyclopédie, 34 (avril). URL: http://www.revues.org/ index $160 . \mathrm{html}$

Lojkine, S. (1999). Le décentrement matérialiste du champ des connaissances dans l'Encyclopédie. Recherches sur Diderot et sur l'Encyclopédie, 26 (avril), 65-84.

Maupertuis, P.-L. Moreau de (1756a [1751]), Essai de cosmologie. In Euvres. T. I (pp. 3-80). Nouv. éd. Lyon : Jean-Marie Bruysset.

Maupertuis, P.-L. Moreau de (1756b [1754]). Système de la nature soit Essai sur la formation des corps organisés, suivi de Réponse aux objections de M. Diderot. In Euvres. T. II (pp. 135-184). Lyon : Jean-Marie Bruysset.

Quintili, P. (2006). La position de la physiologie philosophique de Diderot par rapport au Système des connaissances. Recherches sur Diderot et sur l'Encyclopédie, 40-41, 1-11. URL: http://journals.openedition.org/rde/351

Réaumur, R. A. F. de (1734). Mémoires pour servir à l'histoire des insectes. T. I. Sur les chenilles et sur les papillons. Paris : Imprimerie Royale.

Rey, A.-L. (2003). Le leibnizo-newtonianisme: la construction d'une philosophie naturelle complexe dans la première moitié du $18^{\mathrm{e}}$ siècle. Dix-huitième siècle, 1, 45, 115-129.

Roger, J. (1993). Les sciences de la vie dans la pensée française du XVIII siècle. Nouv. éd. Paris : Albin Michel.

Saint-Amand, P. (1984). Diderot. Le labyrinthe de la relation. Paris : Vrin.

Soubbotnik, M. A. (2010). Interprétation et variation : sur une mise en texte de la connaissance expérimentale chez Diderot. In La mise en texte des savoirs (pp. 165-177). Éds. K. Matsuzawa \& G. Séginger. Strasbourg, Presses Universitaires de Strasbourg.

Vuillemin, N. (2010). Hydres de Lerne et arbres animés: fantasmagories savantes autour du polype. Dix-huitième siècle, 42, 321-338. 